retrospectively analyzed 100 renal core biopsies from 92 patients with incidentally discovered lesions of $<5 \mathrm{~cm}$. Biopsy results were compared to histologic findings obtained at nephrectomy, where available.

Of the 70 biopsies that yielded diagnostic information, almost a third demonstrated benign histology, despite the associated radiologic findings being indicative of malignancya much higher proportion than expected. Furthermore, when biopsy results were compared with histologic findings obtained at nephrectomy, the sensitivity and specificity of biopsy was $100 \%$, for both benign and malignant lesions.

The only major complication observed was one case of post-biopsy hemorrhage. In the light of this risk, the authors suggest implementing a $24 \mathrm{~h}$ observation period following renal biopsy.

The authors conclude that preoperative renal core biopsies should be routinely used in the diagnosis of incidentally detected small renal masses. These biopsies should be interpreted by a specialist urological pathologist.

Original article Vasudevan A et al. (2006) Incidental renal tumours: the frequency of benign lesions and the role of preoperative core biopsy. BJU Int 97: 946-949

\section{Enuresis can improve after surgery for upper-airway obstruction}

Nocturnal enuresis is a common pediatric problem that has been anecdotally linked with obstructive sleep apnea. A new, prospective study undertaken in the US confirms this link, and suggests that obstructive airway disease might have a role in the etiology of enuresis.

Firoozi et al. evaluated 86 consecutive children (age range 3-10 years, 46 boys) with upperairway obstruction who underwent surgery to treat adenotonsillar hyperplasia. Before surgery, $42 \%$ of these children had primary nocturnal enuresis (a much higher rate than in the general population). After surgery, enuresis improved in a third of the children, and completely resolved in another third. Firoozi and colleagues note that roughly twice as many children achieved complete resolution of their enuresis as would be expected without intervention, and suggest that diurnal variation in levels of antidiuretic hormone (secretion of which is normally elevated at night, but might be disrupted by sleep apnea) could be responsible.

The authors also found other changes in voiding after surgery. Surprisingly, across almost all patient groups, there was a substantial decrease in the number of daytime enuresis episodes and in the number of daily voids. This finding is hard to explain: increased daytime voiding might be expected in patients whose nocturnal enuresis resolved. Firoozi et al. propose that upper-airway obstruction might also have a role in daytime enuresis, perhaps because the changes in hormonal milieu that occur after surgery result in increased functional bladder capacity.

Clinicians should be aware that upper-airway obstruction might contribute to enuresis, they say.

Original article Firoozi F et al. (2006) Resolution of diurnal incontinence and nocturnal enuresis after adenotonsillectomy in children. J Urol 175: 1885-1888

\section{PSA nadir after radiotherapy for prostate cancer is a predictor of outcome}

Nadir prostate specific antigen (PSA) levels and time to nadir PSA (TnPSA) following radiotherapy have been proposed as predictors of outcome in prostate cancer. Ray et al. attempted to determine the importance of these parameters as predictors of biochemical and distant recurrence in 4,833 patients who were treated with external beam radiotherapy for prostate cancer at nine US institutions. The endpoints were PSA disease-free survival (PSA-DFS) - classed as freedom from PSA failure as defined by the American Society for Therapeutic Radiology and Oncology, initiation of androgen suppression after radiotherapy, or documented local or distant failure-and distant metastasis-free survival (DMFS).

Lower nadir PSA level and longer TnPSA were associated with increased PSA-DFS. Nadir PSA levels of 0-0.49, 0.50-0.99, 1.00-1.99 and $\geq 2.00 \mathrm{ng} / \mathrm{ml}$ were associated with 8-year PSADFS of $75 \%, 52 \%, 41 \%$ and $18 \%$, respectively $(P<0.0001$ for all), while TnPSAs of 0-5.9, 6.0$11.9,12.0-23.9$ and $\geq 24$ months were associated with PSA-DFS of $27 \%, 32 \%, 42 \%$ and $74 \%$, respectively $(P<0.0001$ for all). Similarly, DMFS correlated positively with TnPSA and inversely with nadir PSA concentration. 